\title{
REW program for the HRTEM image simulation, the alignment and the focus variation image reconstruction
}

\author{
F. Lin,* J.W. Weng, ${ }^{*}$ and J.H. Chen,** \\ * College of Science, South China Agricultural University, Guangzhou 510642, P. R. China \\ ** Center for High-Resolution Electron Microscopy, College of Material Science and Engineering, \\ Hunan University, Changsha Hunan 410082, P. R. China
}

The application program of REW was developed by the $\mathrm{C}++$ programming language on $\mathrm{VC}$ developing environment and could run as a standalone application in any personal computer (PC) with Microsoft Windows operation system [1]. It was developed for users to perform the alignment and the focus variation image reconstruction. In the newest version of above 2.0.00, the HRTEM image could be simulated from a crystal structure and the flexible alignment could be implemented for the through-focus images.

In summary, the REW program consisted of the functions as follows. (1) Image display, filtering, rotation and shift. Specially, image shift and rotation could be performed in the sub-pixel precise. (2) The one-dimension and two-dimension analyses of the contrast transfer function (CTF). The parameters of the spherical aberration, defocus, beam tilt, 2-fold and 3-fold astigmatism, and axial coma were all taken into account. Fig. 1 shows the CTF analyses in REW program. (3) The HRTEM image could be simulated from a pre-built crystal structure via the multi-slice method [2]. In REW program, the crystal structure could be built based on its space group, or read from a PDB file. Fig. 2 shows the structure mode displayed in REW, the simulated HRTEM images, the amplitude map and the phase map of the exit wave. (4) Flexible alignment. The approaches of the general CrossCorrelation Function, the Mutual Correlation Function, the Phase Correlation Function and the Phase-Compensated Phase Correlation Function could be used for aligning the two images or a series of images [3]. Especially, the circular aperture filter of any size could be applied for the correlation map to obtain the accurate alignment. (5) Focus variation image reconstruction. The methods of the maximum-likelihood algorithm and the IWFR algorithm could be utilized in REW [4,5]. Fig. 3(a) shows one of the experimental through-focus images taken in the Cs-corrector TEM and Fig. 3(b) shows the phase map of the reconstructed wave.

REW progrom is completely free and user could get the executing file and helps through the email of flin_rew@163.com [6].

\section{References}

[1] F. Lin, Q. Chen, L.-M. Peng, Journal of Applied Crystallography, 40 (2007) 614.

[2] E.J. Kirkland, Advanced Computing in Electron Microscopy, Plenum Press, New York, 1998.

[3] R.R. Meyer. A.I. Kirkland, W.O. Saxton, Ultramicroscop 92 (2002) 89.

[4] W.M.J. Coene, A. Thust, M. Op de Beeck, D. Van Dyck, Ultramicroscopy 64 (1996) 109.

[5] L.J. Allen, W. McBride, N.L. O'Leary, M.P. Oxley, Ultramicroscopy 100 (2004) 91.

[6] This research was supported by the National Science Foundation of China (grant Nos. 60802003 and 50771043), President Fund of South China Agricultural University (No. 4900K07417), the National Basic Research (973) Program of China (No. 2008CB617608 and No. 2009CB623704). The aid of Prof. L.M. Peng is gratefully acknowledged. 


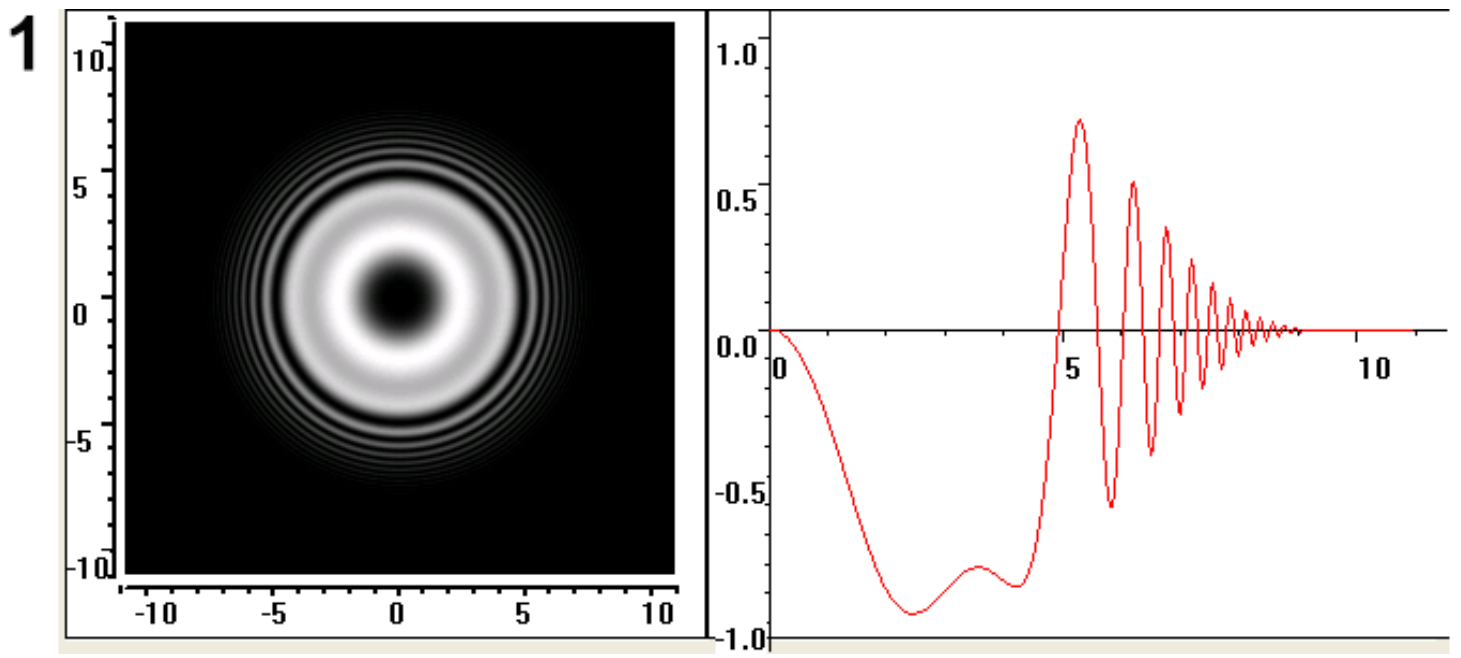

FIG. 1. The CTF analyses in REW program. (a) The 2-dimension analysis of the square of the CTF. (b) The 1-dimnesion analysis of the CTF. The spatial frequency at the $\mathrm{x}$-axis was in the $\mathrm{nm}^{-1}$ units.

2 (a)

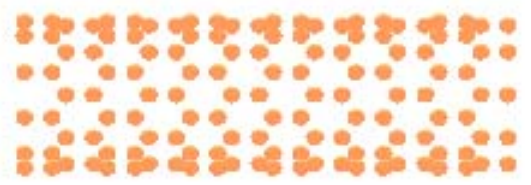

(b)

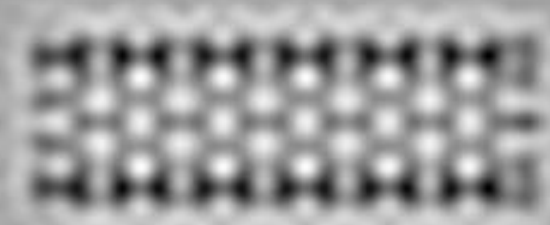

(c)

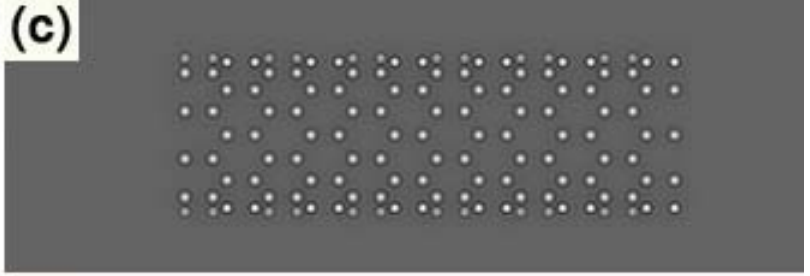

(d)

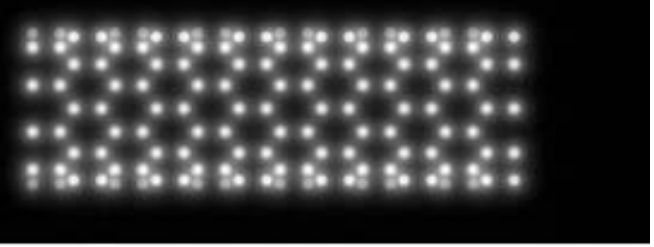

FIG. 2. (a) The projected structure of carbon nano-tube displayed in REW. (b) The simulated HRTEM image. The conditions were: $\mathrm{Cs}=0.6 \mathrm{~mm}$, the acceleration voltage of $300 \mathrm{KV}$ and the Scherzer defocus. (c) The amplitude map of the simulated exit wave. (d) The phase map of the wave.

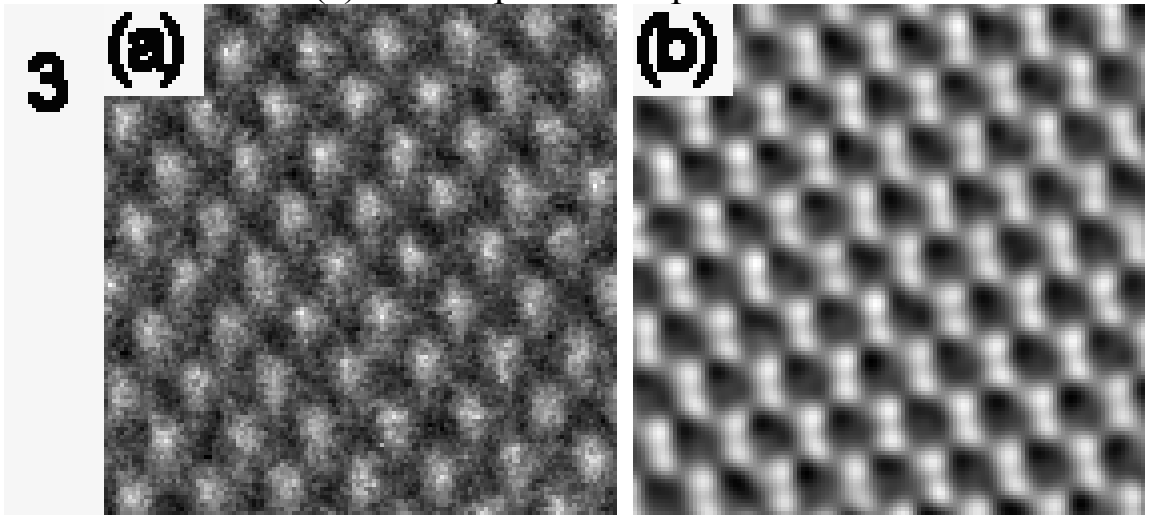

FIG. 3. (a) The experimental image of Si [110], which was taken in a Cs-corrector TEM at the defocus of $-5 \mathrm{~nm}$. (b) The phase map of the exit wave reconstructed from ten experimental images by means of the MAL algorithm. 